\title{
LIFETIME ASYMPTOTICS OF ITERATED BROWNIAN MOTION IN $\mathbb{R}^{n *}$
}

\author{
ERKAN NANE ${ }^{1,2}$
}

\begin{abstract}
Let $\tau_{D}(Z)$ be the first exit time of iterated Brownian motion from a domain $D \subset \mathbb{R}^{n}$ started at $z \in D$ and let $P_{z}\left[\tau_{D}(Z)>t\right]$ be its distribution. In this paper we establish the exact asymptotics of $P_{z}\left[\tau_{D}(Z)>t\right]$ over bounded domains as an improvement of the results in DeBlassie (2004) [12] and Nane (2006) [24], for $z \in D$

$$
\lim _{t \rightarrow \infty} t^{-1 / 2} \exp \left(\frac{3}{2} \pi^{2 / 3} \lambda_{D}^{2 / 3} t^{1 / 3}\right) P_{z}\left[\tau_{D}(Z)>t\right]=C(z)
$$

where $C(z)=\left(\lambda_{D} 2^{7 / 2}\right) / \sqrt{3 \pi}\left(\psi(z) \int_{D} \psi(y) \mathrm{d} y\right)^{2}$. Here $\lambda_{D}$ is the first eigenvalue of the Dirichlet Laplacian $\frac{1}{2} \Delta$ in $D$, and $\psi$ is the eigenfunction corresponding to $\lambda_{D}$. We also study lifetime asymptotics of Brownian-time Brownian motion, $Z_{t}^{1}=z+X(|Y(t)|)$, where $X_{t}$ and $Y_{t}$ are independent onedimensional Brownian motions, in several unbounded domains. Using these results we obtain partial results for lifetime asymptotics of iterated Brownian motion in these unbounded domains.
\end{abstract}

Mathematics Subject Classification. 60J65, 60K99.

Received May 5, 2006. Revised September 8, 2006.

\section{IntRoduction AND STATEMENT OF MAIN RESUlts}

Iterated Brownian motion (IBM) has attracted the interest of several authors $[1-3,7-10,12,15,18,23,24,27,29]$. Several other iterated processes including Brownian-time Brownian motion (BTBM) have also been studied $[1,2,19,25,26]$. One of the main differences between these iterated processes and Brownian motion is that they are not Markov processes. However, these processes have many properties similar to that of Brownian motion (see $[2,3,12,23]$, and references therein).

To define iterated Brownian motion $Z_{t}$ started at $z \in \mathbb{R}$, let $X_{t}^{+}, X_{t}^{-}$and $Y_{t}$ be three independent onedimensional Brownian motions, all started at 0 . Two-sided Brownian motion is defined by

$$
X_{t}= \begin{cases}X_{t}^{+}, & t \geq 0 \\ X_{(-t)}^{-}, & t<0 .\end{cases}
$$

\footnotetext{
Keywords and phrases. Iterated Brownian motion, Brownian-time Brownian motion, exit time, bounded domain, twisted domain, unbounded convex domain.

* Supported in part by NSF Grant \# 9700585-DMS.

1 Department of Mathematics, Purdue University, West Lafayette, IN 47906, USA; enane@math.purdue.edu

2 Current address: Department of Statistics and Probability, Michigan State University, A413 Wells Hall, East Lansing, MI 48824-1027, USA; nane@stt.msu.edu 
Then iterated Brownian motion started at $z \in \mathbb{R}$ is

$$
Z_{t}=z+X\left(Y_{t}\right), \quad t \geq 0 .
$$

In $\mathbb{R}^{n}$, one requires $X^{ \pm}$to be independent $n$-dimensional Brownian motions. This is the version of the iterated Brownian motion due to Burdzy, see [7].

We next define another closely related process, the so called Brownian-time Brownian motion. Let $X_{t}$ and $Y_{t}$ be two independent one-dimensional Brownian motions, all started at 0. Brownian-time Brownian motion started at $z \in \mathbb{R}$ is

$$
Z_{t}^{1}=z+X\left(\left|Y_{t}\right|\right) \quad t \geq 0
$$

In $\mathbb{R}^{n}$ one requires $X$ to be an $n$-dimensional Brownian motion.

Let $\tau_{D}$ be the first exit time of Brownian motion from a domain $D \subset \mathbb{R}^{n}$. The large time behavior of $P_{z}\left[\tau_{D}>t\right]$ has been studied for several types of domains, including general cones [5,11], parabola-shaped domains [4,22], twisted domains [13], unbounded convex domains [21] and bounded domains [28]. Our aim in this article is to do the same for the first exit time of IBM from bounded domains in $\mathbb{R}^{n}$, and for the first exit time of BTBM from several domains in $\mathbb{R}^{n}$. See Bañuelos and DeBlassie [3], Li [21], Lifshits and Shi [22] and Nane [23] for a survey of results obtained for Brownian motion and iterated Brownian motion in these domains.

For many bounded domains $D \subset \mathbb{R}^{n}$ the asymptotics of $P_{z}\left[\tau_{D}>t\right]$ are well-known. (See [28] for a more precise statement of this.) For $z \in D$,

$$
\lim _{t \rightarrow \infty} e^{\lambda_{D} t} P_{z}\left[\tau_{D}>t\right]=\psi(z) \int_{D} \psi(y) \mathrm{d} y,
$$

where $\lambda_{D}$ is the first eigenvalue of $\frac{1}{2} \Delta$ with Dirichlet boundary conditions and $\psi$ is its corresponding eigenfunction.

DeBlassie [12] proved that for iterated Brownian motion in bounded domains, for $z \in D$,

$$
\lim _{t \rightarrow \infty} t^{-1 / 3} \log P_{z}\left[\tau_{D}(Z)>t\right]=-\frac{3}{2} \pi^{2 / 3} \lambda_{D}^{2 / 3}
$$

The limits (1.1) and (1.2) are very different in that the latter involves taking the logarithm which may kill many unwanted multipliers of the exponential. It is then natural to ask if it is possible to obtain an analogue of (1.1) for IBM. That is, can one remove the log in (1.2)? In [24], we improved the limit in (1.2) as follows, for $z \in D$,

$$
\begin{aligned}
2 C(z) & \leq \liminf _{t \rightarrow \infty} t^{-1 / 2} \exp \left(\frac{3}{2} \pi^{2 / 3} \lambda_{D}^{2 / 3} t^{1 / 3}\right) P_{z}\left[\tau_{D}(Z)>t\right] \\
& \leq \limsup _{t \rightarrow \infty} t^{-1 / 2} \exp \left(\frac{3}{2} \pi^{2 / 3} \lambda_{D}^{2 / 3} t^{1 / 3}\right) P_{z}\left[\tau_{D}(Z)>t\right] \leq \pi C(z),
\end{aligned}
$$

where $C(z)=\lambda_{D} \sqrt{2 \pi / 3}\left(\psi(z) \int_{D} \psi(y) \mathrm{d} y\right)^{2}$.

In this paper we prove the following theorem which improves both limits above.

Theorem 1.1. Let $D \subset \mathbb{R}^{n}$ be a domain for which (1.1) holds pointwise and let $\lambda_{D}$ and $\psi$ be as above. Then for $z \in D$,

$$
\lim _{t \rightarrow \infty} t^{-1 / 2} \exp \left(\frac{3}{2} \pi^{2 / 3} \lambda_{D}^{2 / 3} t^{1 / 3}\right) P_{z}\left[\tau_{D}(Z)>t\right]=\frac{\left(\lambda_{D} 2^{7 / 2}\right)}{\sqrt{3 \pi}}\left(\psi(z) \int_{D} \psi(y) \mathrm{d} y\right)^{2} .
$$

Remark 1. Observe that $2 \lambda_{D} \sqrt{2 \pi / 3} \leq\left(\lambda_{D} 2^{7 / 2}\right) / \sqrt{3 \pi} \leq \pi \lambda_{D} \sqrt{2 \pi / 3}$, so Theorem 1.1 is in agreement with the results obtained previously in $[12,24]$. 
In [13], DeBlassie and Smits studied the tail behavior of the first exit time of Brownian motion in twisted domains in the plane. Let $D \subset \mathbb{R}^{2}$ be a domain whose boundary consists of three curves (in polar coordinates)

$$
\begin{array}{ll}
C_{1}: \quad \theta=f_{1}(r), \quad r \geq r_{1} \\
C_{2}: \quad \theta=f_{2}(r), \quad r \geq r_{1} \\
C_{3}: \quad r=r_{1}, \quad f_{2}(r) \leq \theta \leq f_{1}(r)
\end{array}
$$

where $f_{1}$ and $f_{2}$ are smooth and the curves $C_{1}$ and $C_{2}$ do not cross:

$$
0<f_{1}(r)-f_{2}(r)<\pi, \quad r \geq r_{1} .
$$

DeBlassie and Smits call $D$ a twisted domain if there are constants $r_{0}>0, \gamma>0$ and $p \in(0,1]$ and a smooth function $f(r)$ such that the curves $f_{1}(r)$ and $f_{2}(r), r \geq r_{0}$, are obtained from $f(r)$ by moving out $\pm \gamma r^{p}$ units along the normal to the curve $\theta=f(r)$ at the point whose polar coordinates are $(r, f(r))$. They call $\gamma r^{p}$ the growth radius and $\theta=f(r)$ the generating curve. DeBlassie and Smits [13] (Th. 1.1) have the following tail behavior of the first exit time of Brownian motion in twisted domains $D \subset \mathbb{R}^{2}$ with growth radius $\gamma r^{p}, \gamma>0$, $0<p<1$

where

$$
\lim _{t \rightarrow \infty} t^{-\left(\frac{1-p}{1+p}\right)} \log P_{z}\left[\tau_{D}>t\right]=-l_{1}=-\left[\frac{\pi^{2 p-1}}{\gamma 2^{2 p}(1-p)^{2 p}}\right]^{\frac{2}{p+1}} C_{p}
$$

$$
C_{p}=(1+p)\left[\frac{\pi^{2+p}}{8^{p} p^{2 p}(1-p)^{1-p}} \frac{\Gamma^{2 p}\left(\frac{1-p}{2 p}\right)}{\Gamma^{2 p}\left(\frac{1}{2 p}\right)}\right]^{\frac{1}{p+1}} .
$$

For these domains, Nane [23] obtained the following result for IBM: for all $z \in D$,

$$
\lim _{t \rightarrow \infty} t^{-\left(\frac{1-p}{3+p}\right)} \log P_{z}\left[\tau_{D}(Z)>t\right]=-\left(\frac{3+p}{2+2 p}\right)\left(\frac{1+p}{1-p}\right)^{\left(\frac{1-p}{3+p}\right)} \pi^{\left(\frac{2-2 p}{3+p}\right)} l_{1}^{\left(\frac{2+2 p}{3+p}\right)},
$$

where $l_{1}$ is the limit given by (1.3).

We obtained in [24], the following for BTBM in twisted domains, for $z \in D$,

$$
\lim _{t \rightarrow \infty} t^{-\left(\frac{1-p}{p+3}\right)} \log P_{z}\left[\tau_{D}\left(Z^{1}\right)>t\right]=-2^{\left(\frac{2 p-2}{3+p}\right)}\left(\frac{3+p}{2+2 p}\right)\left(\frac{1+p}{1-p}\right)^{\left(\frac{1-p}{3+p}\right)} \pi^{\left(\frac{2-2 p}{3+p}\right)} l_{1}^{\left(\frac{2+2 p}{3+p}\right)},
$$

where $l_{1}$ is the limit given by the limit given by (1.3).

DeBlassie and Smits [13] also obtained similar results for $p=1$. Let $D \subset \mathbb{R}^{2}$ be a twisted domain with growth radius $\gamma r, \gamma>0$. Then for $z \in D$,

$$
\lim _{t \rightarrow \infty}[\log t]^{-1} \log P_{z}\left[\tau_{D}>t\right]=-C(\gamma)=-\pi\left[4 \arccos \frac{1}{\sqrt{1+\gamma^{2}}}\right]^{-1} .
$$

We obtain the following lifetime asymptotics of BTBM in twisted domains.

Theorem 1.2. Let $D \subset \mathbb{R}^{2}$ be a twisted domain with growth radius $\gamma r, \gamma>0$. Then for $z \in D$,

$$
\lim _{t \rightarrow \infty}[\log t]^{-1} \log P_{z}\left[\tau_{D}\left(Z^{1}\right)>t\right]=-C(\gamma) / 2
$$

with $C(\gamma)$ as in (1.4). 
Using Theorem 1.3. from [24], which says that for all $z \in D$ and all $t>0$,

$$
P_{z}\left[\tau_{D}(Z)>t\right] \leq 2 P_{z}\left[\tau_{D}\left(Z^{1}\right)>t\right]
$$

we obtain the following for IBM in twisted domains.

Corollary 1.1. Let $D \subset \mathbb{R}^{2}$ be a twisted domain with growth radius $\gamma r, \gamma>0$. Then for $z \in D$,

$$
\limsup _{t \rightarrow \infty}[\log t]^{-1} \log P_{z}\left[\tau_{D}(Z)>t\right] \leq-C(\gamma) / 2,
$$

with $C(\gamma)$ as in (1.4).

In [21], using Gaussian techniques, Li studied lifetime asymptotics of Brownian motion in domains of the following form

$$
P_{f}=\left\{(x, y) \in \mathbb{R}^{n+1}: y>f(x), x \in \mathbb{R}^{n}\right\}
$$

for $f(x)=\exp \left(|x|^{p}\right), p>0$. Li established that for $z \in P_{f}$,

$$
\lim _{t \rightarrow \infty} t^{-1}(\log t)^{2 / p} \log P_{z}\left[\tau_{P_{f}}>t\right]=-j_{\nu}^{2},
$$

where $\nu=(n-2) / 2$ and $j_{\nu}$ is the smallest positive zero of the Bessel function $J_{v}$.

We obtain the following theorem in these domains

Theorem 1.3. Let $P_{f}$ be as above with $f(x)=\exp \left(|x|^{p}\right), p>0$. Then for $z \in P_{f}$,

$$
\lim _{t \rightarrow \infty} t^{-1 / 3}(\log t)^{4 / 3 p} \log P_{z}\left[\tau_{P_{f}}\left(Z^{1}\right)>t\right]=-C(p),
$$

where

$$
C(p)=(3 / 2)^{(4+3 p) / 3 p} 2^{1 / 3}\left(j_{\nu}^{2} 2^{2 / p}\right)^{2 / 3}\left(\pi^{2} / 8\right)^{1 / 3} .
$$

Using Theorem 1.3. from [24], we obtain the following for IBM in these domains.

Corollary 1.2. Let $P_{f}$ be as above with $f(x)=\exp \left(|x|^{p}\right), p>0$. Then for $z \in P_{f}$,

$$
\limsup _{t \rightarrow \infty} t^{-1 / 3}(\log t)^{4 / 3 p} \log P_{z}\left[\tau_{P_{f}}(Z)>t\right] \leq-C(p) .
$$

For $f(x)=h(|x|)$ and $h^{-1}(x)=A x^{\alpha}(\log x)^{\beta}, x>1$. Li obtained the following: let $\epsilon>0$. For $t$ large, $z \in P_{f}$

$$
-(1+\epsilon) C_{\alpha, \beta, 1} \leq t^{-\frac{(1-\alpha)}{(1+\alpha)}}(\log t)^{\frac{2 \beta}{(1+\alpha)}} \log P_{z}\left[\tau_{P_{f}}>t\right] \leq-(1-\epsilon) C_{\alpha, \beta, 2},
$$

where

and

$$
C_{\alpha, \beta, 1}=2^{-1}(1-\alpha)^{-1}\left(\alpha^{-\alpha}(1+\alpha)^{2 \beta+2} A^{-2} j_{\nu}^{2}\right) 1 /(1+\alpha)
$$

where $C=(1-\alpha)^{-1} 2^{2 \beta-1} A^{-2} j_{\nu}^{2}$.

$$
\left.C_{\alpha, \beta, 2}=(1+\alpha)(2 \alpha)^{-\alpha /(1+\alpha)}\left(2^{-1}(1+\alpha)\right)^{2 \beta /(1+\alpha)}\right) C^{1 /(1+\alpha)}
$$

We have the following for BTBM in these domains.

Theorem 1.4. For $0<\alpha<1$ and $\beta \in \mathbb{R}$,

$$
\begin{aligned}
-C(1) & \leq \liminf _{t \rightarrow \infty} t^{-(1-\alpha) /(3+\alpha)}(\log t)^{4 \beta(1+\alpha) /(3+\alpha)} \log P_{z}\left[\tau_{P_{f}}\left(Z^{1}\right)>t\right] \\
& \leq \limsup _{t \rightarrow \infty} t^{-(1-\alpha) /(3 \alpha+1)}(\log t)^{4 \beta(1+\alpha) /(3+\alpha)} \log P_{z}\left[\tau_{P_{f}}\left(Z^{1}\right)>t\right] \\
& \leq-C(2)
\end{aligned}
$$


where

and

$$
C(1)=\left(\frac{3+\alpha}{2(1+\alpha)}\right)^{\frac{3+\alpha+4 \beta}{3+\alpha}}\left(\frac{1-\alpha}{(3+\alpha)}\right)\left(\pi^{2} / 8\right)^{\frac{1-\alpha}{(3+\alpha)}}\left(C_{\alpha, \beta, 1}\right)^{\frac{2(1+\alpha)}{(3+\alpha)}} 2^{\frac{4 \beta}{(3+\alpha)}}
$$

$$
C(2)=\left(\frac{3+\alpha}{2(1+\alpha)}\right)^{\frac{3+\alpha+4 \beta}{3+\alpha}}\left(\frac{1-\alpha}{(3+\alpha)}\right)\left(\pi^{2} / 8\right)^{\frac{1-\alpha}{(3+\alpha)}}\left(C_{\alpha, \beta, 2}\right)^{\frac{2(1+\alpha)}{(3+\alpha)}} 2^{\frac{4 \beta}{(3+\alpha)}} .
$$

Using Theorem 1.3. from [24], we obtain the following for IBM in these domains.

Corollary 1.3. For $0<\alpha<1$ and $\beta \in \mathbb{R}$. Let $P_{f}$ be as above with $f(x)=h(|x|), h^{-1}(x)=A x^{\alpha}(\log x)^{\beta}$, $x>1$. Then for $z \in P_{f}$,

$$
\limsup _{t \rightarrow \infty} t^{-(1-\alpha) /(3 \alpha+1)}(\log t)^{4 \beta(1+\alpha) /(3+\alpha)} \log P_{z}\left[\tau_{P_{f}}\left(Z^{1}\right)>t\right] \leq-C(2)
$$

where $C(2)$ is as above.

The paper is organized as follows. In Section 2, we give some preliminary lemmas to be used in the proof of main results. We also recall several asymptotic results from Nane [24] to be used in the proof of main results. Theorem 1.1 is proved in Section 3. Section 4 is devoted to prove Theorems 1.2, 1.3 and 1.4.

\section{Preliminaries}

In this section we state some preliminary facts that will be used in the proof of main results.

In what follows we will write $f \approx g$ and $f \lesssim g$ to mean that for some positive $C_{1}$ and $C_{2}, C_{1} \leq f / g \leq C_{2}$ and $f \leq C_{1} g$, respectively. We will also write $f(t) \sim g(t)$, as $t \rightarrow \infty$, to mean that $f(t) / g(t) \rightarrow 1$, as $t \rightarrow \infty$.

The main fact is the following Tauberian theorem ([14, Laplace transform method, 1958, Chapter 4]). Laporte $[20]$ also studied these types of integrals. Let $h$ and $f$ be continuous functions on $\mathbb{R}$. Suppose $f$ is non-positive and has a global max at $x_{0}, f^{\prime}\left(x_{0}\right)=0, f^{\prime \prime}\left(x_{0}\right)<0$ and $h\left(x_{0}\right) \neq 0$ and $\int_{-\infty}^{\infty} h(x) \exp (\lambda f(x))<\infty$ for all $\lambda>0$. Then as $\lambda \rightarrow \infty$,

$$
\int_{0}^{\infty} h(x) \exp (\lambda f(x)) \mathrm{d} x \sim h\left(x_{0}\right) \exp \left(\lambda f\left(x_{0}\right)\right) \sqrt{\frac{2 \pi}{\lambda\left|f^{\prime \prime}\left(x_{0}\right)\right|}} .
$$

It can be easily seen from this that as $\lambda \rightarrow \infty$,

$$
\int_{0}^{\infty} \exp \left(-\lambda\left(x+x^{-2}\right)\right) \mathrm{d} x \sim \exp \left(-3 \lambda 2^{-2 / 3}\right) \sqrt{\frac{2^{4 / 3} \pi}{3 \lambda}} .
$$

Similarly, as $t \rightarrow \infty$,

$$
\int_{0}^{\infty} \exp \left(-\frac{a t}{u^{2}}-b u\right) \mathrm{d} u \sim \sqrt{\frac{\pi}{3}} 2^{2 / 3} a^{1 / 6} b^{-2 / 3} t^{1 / 6} \exp \left(-3 a^{1 / 3} b^{2 / 3} 2^{-2 / 3} t^{1 / 3}\right) .
$$

This follows from equation (2.2) after making the change of variables $u=\left(a t b^{-1}\right)^{1 / 3} x$.

Finally, we obtain, as $t \rightarrow \infty$,

$$
\int_{0}^{\infty} u \exp \left(-\frac{a t}{u^{2}}-b u\right) \mathrm{d} u \sim 2 \sqrt{\frac{\pi}{3}} a^{1 / 2} b^{-1} t^{1 / 2} \exp \left(-3 a^{1 / 3} b^{2 / 3} 2^{-2 / 3} t^{1 / 3}\right) .
$$

Writing the power series for the cosine function we easily see that as $t \rightarrow \infty$,

$$
\int_{0}^{\infty} x \cos (\pi K / x) \exp \left(-\frac{\pi^{2} t}{2 x^{2}}-\lambda_{D} x\right) \mathrm{d} x \sim 2 \sqrt{\frac{\pi}{3}}\left(\frac{\pi^{2}}{2}\right)^{1 / 2} \lambda_{D}^{-1} t^{1 / 2} \exp \left(-\frac{3}{2} \pi^{2 / 3} \lambda_{D}^{2 / 3} t^{1 / 3}\right) .
$$


We next state a version of de Bruijn's Tauberian Theorem (Kasahara [17] Th. 3 and Bingham, Goldie and Teugels [6] p. 254).

Theorem 2.1 (de Bruijn's Tauberian Theorem). Let $\xi$ be a positive random variable. Then, for $\alpha>0$ and $\beta \in \mathbb{R}$

$$
\log P[\xi \leq \epsilon] \sim-C \epsilon^{-\alpha}|\log \epsilon|^{\beta} \quad \text { as } \quad \epsilon \rightarrow 0^{+}
$$

if and only if

$$
\log E[\exp (-\lambda \xi)] \sim-(1+\alpha)^{1-\beta /(1+\alpha)} \alpha^{-\alpha /(1+\alpha)} C^{1 /(1+\alpha)} \lambda^{\alpha /(1+\alpha)}(\log \lambda)^{\beta /(1+\alpha)}
$$

as $\lambda \rightarrow \infty$.

In the case $\alpha=0$, if $x \rightarrow 0^{+}$

$$
[|\log x|]^{-1} \log P[\xi \leq x] \sim-c / 2
$$

Then as $\lambda \rightarrow \infty$

$$
[\log \lambda]^{-1} \log E[\exp (-\lambda \xi)] \sim-c / 2 .
$$

Next, we will recall some lemmas that will used in Sections 3 and 4. The following lemma is proved in [12] Lemma A1 (it also follows from more general results on "intrinsic ultracontractivity"). We include it for completeness.

For $I \subset \mathbb{R}$ an open interval, we write

$$
\eta_{I}=\eta(I)=\inf \left\{t \geq 0: \quad Y_{t} \notin I\right\} .
$$

Lemma 2.1. As $t \rightarrow \infty$,

$$
P_{x}\left[\eta_{(0,1)}>t\right] \sim \frac{4}{\pi} e^{-\frac{\pi^{2} t}{2}} \sin \pi x, \text { uniformly for } x \in(0,1) .
$$

We recall a result from Nane [23] Lemma 6.2, that will be used for the process $Z^{1}$.

Lemma 2.2. Let $B=\left\{u>0: t / u^{2}>M\right\}$ for $M$ large. Then on $B$,

$$
\frac{\mathrm{d}}{\mathrm{d} u} P_{0}\left[\eta_{(-u, u)}>t\right] \sim \exp \left(-\frac{\pi^{2} t}{8 u^{2}}\right) \frac{\pi t}{u^{3}} .
$$

\section{ITERATED BROWNIAN MOTION IN BOUNDED DOMAINS}

If $D \subset \mathbb{R}^{n}$ is an open set, write

$$
\tau_{D}^{ \pm}(z)=\inf \left\{t \geq 0: \quad X_{t}^{ \pm}+z \notin D\right\},
$$

and if $I \subset \mathbb{R}$ is an open interval, write

$$
\eta_{I}=\eta(I)=\inf \left\{t \geq 0: \quad Y_{t} \notin I\right\} .
$$

Recall that $\tau_{D}(Z)$ stands for the first exit time of iterated Brownian motion from $D$. As in DeBlassie $[12, \S 3$.], we have by the continuity of the paths for $Z_{t}=z+X\left(Y_{t}\right)$, if $f$ is the probability density of $\tau_{D}^{ \pm}(z)$

$$
P_{z}\left[\tau_{D}(Z)>t\right]=\int_{0}^{\infty} \int_{0}^{\infty} P_{0}\left[\eta_{(-u, v)}>t\right] f(u) f(v) \mathrm{d} v \mathrm{~d} u .
$$


The proof of Theorem 1.1. The following is well-known

$$
P_{0}\left[\eta_{(-u, v)}>t\right]=\frac{4}{\pi} \sum_{n=0}^{\infty} \frac{1}{2 n+1} \exp \left(-\frac{(2 n+1)^{2} \pi^{2}}{2(u+v)^{2}} t\right) \sin \frac{(2 n+1) \pi u}{u+v}
$$

(see Feller [16] pp. 340-342).

Let $\epsilon>0$. From Lemma 2.1, choose $M>0$ so large that

$$
(1-\epsilon) \frac{4}{\pi} \mathrm{e}^{-\frac{\pi^{2} t}{2}} \sin \pi x \leq P_{x}\left[\eta_{(0,1)}>t\right] \leq(1+\epsilon) \frac{4}{\pi} \mathrm{e}^{-\frac{\pi^{2} t}{2}} \sin \pi x,
$$

for $t \geq M$, uniformly $x \in(0,1)$. For a bounded domain with regular boundary it is well-known (see [28] pp. 121-127) that there exists an increasing sequence of eigenvalues, $\lambda_{1}<\lambda_{2} \leq \lambda_{3} \cdots$, and eigenfunctions $\psi_{k}$ corresponding to $\lambda_{k}$ such that,

$$
P_{z}\left[\tau_{D} \leq t\right]=\sum_{k=1}^{\infty} \exp \left(-\lambda_{k} t\right) \psi_{k}(z) \int_{D} \psi_{k}(y) \mathrm{d} y
$$

From the arguments in DeBlassie [12] Lemma A.4

$$
f(t)=\frac{\mathrm{d}}{\mathrm{d} t} P_{z}\left[\tau_{D} \leq t\right]=\sum_{k=1}^{\infty} \lambda_{k} \exp \left(-\lambda_{k} t\right) \psi_{k}(z) \int_{D} \psi_{k}(y) \mathrm{d} y
$$

Finally choose $K>0$ so large that

$$
A(z)(1-\epsilon) \exp \left(-\lambda_{D} u\right) \leq f(u) \leq A(z)(1+\epsilon) \exp \left(-\lambda_{D} u\right)
$$

for all $u \geq K$, where

$$
A(z)=\lambda_{1} \psi_{1}(z) \int_{D} \psi_{1}(y) \mathrm{d} y=\lambda_{D} \psi(z) \int_{D} \psi(y) \mathrm{d} y
$$

We further assume that $t$ is so large that $K<\frac{1}{2} \sqrt{t / M}$. Define $A$ for $K>0$ and $M>0$ as

$$
A=\left\{(u, v): \quad K \leq u \leq \frac{1}{2} \sqrt{\frac{t}{M}}, u \leq v \leq \sqrt{\frac{t}{M}}-u\right\}
$$

By equation (3.3) and from equation (3.10) in [12],

$$
\begin{gathered}
P_{z}\left[\tau_{D}(Z)>t\right]=2 \int_{0}^{\infty} \int_{u}^{\infty} P_{\frac{u}{u+v}}\left[\eta_{(0,1)}>\frac{t}{(u+v)^{2}}\right] f(u) f(v) \mathrm{d} v \mathrm{~d} u \\
\geq C^{1} \int_{K}^{\frac{1}{2} \sqrt{t / M}} \int_{u}^{\sqrt{t / M}-u} \sin \left(\frac{\pi u}{(u+v)}\right) \exp \left(-\frac{\pi^{2} t}{2(u+v)^{2}}\right) \exp \left(-\lambda_{D}(u+v)\right) \mathrm{d} v \mathrm{~d} u
\end{gathered}
$$

where $C^{1}=C^{1}(z)=2(4 / \pi) A(z)^{2}(1-\epsilon)^{3}$. Changing the variables $x=u+v, z=u$ the integral is

$$
=C^{1} \int_{K}^{\frac{1}{2} \sqrt{t / M}} \int_{2 z}^{\sqrt{t / M}} \sin \left(\frac{\pi z}{x}\right) \exp \left(-\frac{\pi^{2} t}{2 x^{2}}\right) \exp \left(-\lambda_{D} x\right) \mathrm{d} x \mathrm{~d} z,
$$


and reversing the order of integration

$$
\begin{aligned}
& =C^{1} \int_{2 K}^{\sqrt{t / M}} \int_{K}^{\frac{1}{2} x} \sin \left(\frac{\pi z}{x}\right) \exp \left(-\frac{\pi^{2} t}{2 x^{2}}\right) \exp \left(-\lambda_{D} x\right) \mathrm{d} z \mathrm{~d} x \\
& =C^{1} / \pi \int_{2 K}^{\sqrt{t / M}} x \cos \left(\frac{\pi K}{x}\right) \exp \left(-\frac{\pi^{2} t}{2 x^{2}}\right) \exp \left(-\lambda_{D} x\right) \mathrm{d} x
\end{aligned}
$$

From equation (2.5) as $t \rightarrow \infty$,

$$
\int_{0}^{\infty} x \cos \left(\frac{\pi K}{x}\right) \exp \left(-\frac{\pi^{2} t}{2 x^{2}}\right) \exp \left(-\lambda_{D} x\right) \mathrm{d} x \sim 2 \sqrt{\frac{\pi}{3}}\left(\frac{\pi^{2}}{2}\right)^{1 / 2} \lambda_{D}^{-1} t^{1 / 2} \exp \left(-\frac{3}{2} \pi^{2 / 3} \lambda_{D}^{2 / 3} t^{1 / 3}\right)
$$

Now for some $c_{1}>0$,

$$
\int_{0}^{2 K} x \exp \left(-\frac{\pi^{2} t}{2 x^{2}}-\lambda_{D} x\right) \mathrm{d} x \leq \mathrm{e}^{-\pi^{2} t / 2 K^{2}} \int_{0}^{2 K} x \exp \left(-\lambda_{D} x\right) \mathrm{d} x \lesssim \mathrm{e}^{-c_{1} t},
$$

and

$$
\int_{\sqrt{t / M}}^{\infty} x \exp \left(-\frac{\pi^{2} t}{2 x^{2}}\right) \exp \left(-\lambda_{D} x\right) \mathrm{d} x \leq \int_{\sqrt{t / M}}^{\infty} x \exp \left(-\lambda_{D} x\right) \mathrm{d} x=\left(\sqrt{t / M} \lambda_{D}^{-1}+\lambda_{D}^{-2}\right) \exp \left(-\lambda_{D} \sqrt{t / M}\right)
$$

Now from equations (3.6)-(3.8) we get

$$
\liminf _{t \rightarrow \infty} t^{-1 / 2} \exp \left(\frac{3}{2} \pi^{2 / 3} \lambda_{D}^{2 / 3} t^{1 / 3}\right) P_{z}\left[\tau_{D}(Z)>t\right] \geq\left(C^{1} / \pi\right) 2 \sqrt{\frac{\pi}{3}}\left(\frac{\pi^{2}}{2}\right)^{1 / 2} \lambda_{D}^{-1} .
$$

For the upper bound for $P\left[\tau_{D}(Z)>t\right]$ from equation (3.10) in [12],

$$
P_{z}\left[\tau_{D}(Z)>t\right]=2 \int_{0}^{\infty} \int_{u}^{\infty} P_{\frac{u}{u+v}}\left[\eta_{(0,1)}>\frac{t}{\left(u+v^{2}\right)}\right] f(u) f(v) \mathrm{d} v \mathrm{~d} u
$$

We define the following sets that make up the domain of integration,

$$
\begin{aligned}
& A_{1}=\{(u, v): v \geq u \geq 0, u+v \geq \sqrt{t / M}\}, \\
& A_{2}=\{(u, v): u \geq 0, v \geq K, u \leq v, u+v \leq \sqrt{t / M}\}, \\
& A_{3}=\{(u, v): 0 \leq u \leq v \leq K\} .
\end{aligned}
$$

Over the set $A_{1}$ we have for some $c>0$,

$$
\iint_{A_{1}} P_{\frac{u}{u+v}}\left[\eta_{(0,1)}>\frac{t}{(u+v)^{2}}\right] f(u) f(v) \mathrm{d} v \mathrm{~d} u \leq \iint_{A_{1}} f(u) f(v) \mathrm{d} v \mathrm{~d} u \leq \exp (-c \sqrt{t / M}) .
$$

The equation (3.11) follows from the distribution of $\tau_{D}$ from equation (3.5). 
Since on $A_{3}, t /(u+v)^{2} \geq M$,

$$
\begin{aligned}
& \iint_{A_{3}} P_{\frac{u}{u+v}}\left[\eta_{(0,1)}>\frac{t}{(u+v)^{2}}\right] f(u) f(v) \mathrm{d} v \mathrm{~d} u \\
& \lesssim \int_{0}^{K} \int_{0}^{K} \exp \left(-\frac{\pi^{2} t}{2(u+v)^{2}}\right) f(u) f(v) \mathrm{d} v \mathrm{~d} u \\
& \leq \exp \left(-\frac{\pi^{2} t}{8 K^{2}}\right) \int_{0}^{K} \int_{0}^{K} f(u) f(v) \mathrm{d} v \mathrm{~d} u \leq \exp \left(-\frac{\pi^{2} t}{8 K^{2}}\right) .
\end{aligned}
$$

Let $C_{1}=C_{1}(z)=2(4 / \pi) A(z)^{2}(1+\epsilon)^{3}$. For the integral over $A_{2}$ we get,

$$
\begin{aligned}
& \iint_{A_{2}} P_{\frac{u}{u+v}}\left[\eta_{(0,1)}>\frac{t}{(u+v)^{2}}\right] f(u) f(v) \mathrm{d} v \mathrm{~d} u \\
\leq & C_{11} \int_{0}^{K} \int_{K}^{\sqrt{t / M}-u} f(u) \exp \left(-\frac{\pi^{2} t}{2(u+v)^{2}}-\lambda_{D} v\right) \mathrm{d} v \mathrm{~d} u \\
+ & C_{1} \int_{K}^{1 / 2 \sqrt{t / M}} \int_{u}^{\sqrt{t / M}-u} \sin \left(\frac{\pi u}{u+v}\right) \exp \left(-\frac{\pi^{2} t}{2(u+v)^{2}}-\lambda_{D}(u+v)\right) \mathrm{d} v \mathrm{~d} u \\
= & I+I I .
\end{aligned}
$$

Changing variables $u+v=z, u=w$

$$
\begin{aligned}
I & \approx \int_{0}^{K} \int_{K}^{\sqrt{t / M}-u} \exp \left(-\frac{\pi^{2} t}{2(u+v)^{2}}\right) f(u) \exp \left(-\lambda_{D} v\right) \mathrm{d} v \mathrm{~d} u \\
& \leq \int_{0}^{K} \int_{w+K}^{\sqrt{t / M}} \exp \left(-\frac{\pi^{2} t}{2 z^{2}}\right) f(w) \exp \left(-\lambda_{D} z\right) \exp \left(\lambda_{D} w\right) \mathrm{d} z \mathrm{~d} w \\
& \leq \exp \left(\lambda_{D} K\right) \int_{0}^{K} f(w) \mathrm{d} w \int_{0}^{\infty} \exp \left(-\frac{\pi^{2} t}{2 z^{2}}\right) \exp \left(-\lambda_{D} z\right) \mathrm{d} z \\
& \lesssim t^{1 / 6} \exp \left(-\frac{3}{2} \pi^{2 / 3} \lambda_{D}^{2 / 3} t^{1 / 3}\right) .
\end{aligned}
$$

Equation (3.14) follows from equation (2.3), with $a=\pi^{2} / 2, b=\lambda_{D}$.

Changing variables $u+v=z, u=w$

$$
\begin{aligned}
I I & \leq C_{1} \int_{K}^{1 / 2 \sqrt{t / M}} \int_{2 w}^{\sqrt{t / M}} \sin \left(\frac{\pi w}{z}\right) \exp \left(-\frac{\pi^{2} t}{2 z^{2}}-\lambda_{D} z\right) \mathrm{d} z \mathrm{~d} w \\
& =C_{1} \int_{2 K}^{\sqrt{t / M}} \int_{K}^{z / 2} \sin \left(\frac{\pi w}{z}\right) \exp \left(-\frac{\pi^{2} t}{2 z^{2}}-\lambda_{D} z\right) \mathrm{d} w \mathrm{~d} z \\
& \leq C_{1} / \pi \int_{2 K}^{\sqrt{t / M}} z \cos \left(\frac{\pi K}{z}\right) \exp \left(-\frac{\pi^{2} t}{2 z^{2}}-\lambda_{D} z\right) \mathrm{d} z \\
& \leq(1+\epsilon)\left(C_{1} / \pi\right) 2 \sqrt{\frac{\pi}{3}}\left(\frac{\pi^{2}}{2}\right)^{1 / 2} \lambda_{D}^{-1} t^{1 / 2} \exp \left(-\frac{3}{2} \pi^{2 / 3} \lambda_{D}^{2 / 3} t^{1 / 3}\right) .
\end{aligned}
$$

Equation (3.15) follows by changing the order of the integration. Equation (3.16) follows from equation (2.5). 
Now from equations (3.11), (3.12), (3.14) and (3.16) we obtain

$$
\limsup _{t \rightarrow \infty} t^{-1 / 2} \exp \left(\frac{3}{2} \pi^{2 / 3} \lambda_{D}^{2 / 3} t^{1 / 3}\right) P_{z}\left[\tau_{D}(Z)>t\right] \leq(1+\epsilon)\left(\frac{C_{1}}{\pi}\right) 2 \sqrt{\frac{\pi}{3}}\left(\frac{\pi^{2}}{2}\right)^{1 / 2} \lambda_{D}^{-1} .
$$

Finally, from equations (3.9) and (3.17) and letting $\epsilon \rightarrow 0$,

$$
\begin{aligned}
C(z) & \leq \liminf _{t \rightarrow \infty} t^{-1 / 2} \exp \left(\frac{3}{2} \pi^{2 / 3} \lambda_{D}^{2 / 3} t^{1 / 3}\right) P_{z}\left[\tau_{D}(Z)>t\right] \\
& \leq \limsup _{t \rightarrow \infty} t^{-1 / 2} \exp \left(\frac{3}{2} \pi^{2 / 3} \lambda_{D}^{2 / 3} t^{1 / 3}\right) P_{z}\left[\tau_{D}(Z)>t\right] \leq C(z),
\end{aligned}
$$

where $C(z)=\left(\lambda_{D} 2^{7 / 2}\right) / \sqrt{3 \pi}\left(\psi(z) \int_{D} \psi(y) \mathrm{d} y\right)^{2}$.

\section{Brownian-time Brownian motion in unbounded DOMAINS}

In this section we study Brownian-time Brownian motion (BTBM), $Z_{t}^{1}$ started at $z \in \mathbb{R}$, in several unbounded domains.

If $D \subset \mathbb{R}^{n}$ is an open set, write

$$
\tau_{D}(z)=\inf \left\{t \geq 0: X_{t}+z \notin D\right\},
$$

and if $I \subset \mathbb{R}$ is an open interval, we write

$$
\eta_{I}=\inf \left\{t \geq 0: Y_{t} \notin I\right\} .
$$

Let $\tau_{D}\left(Z^{1}\right)$ stand for the first exit time of BTBM from $D$. We have by the continuity of paths

$$
P_{z}\left[\tau_{D}\left(Z^{1}\right)>t\right]=P\left[\eta\left(-\tau_{D}(z), \tau_{D}(z)\right)>t\right] .
$$

Proof of Theorem 1.2. Let $\epsilon>0$. From Lemma 2.2, choose $M>0$ so large that

$$
(1-\epsilon) \exp \left(-\frac{\pi^{2} t}{8 u^{2}}\right) \frac{\pi t}{u^{3}} \leq \frac{\mathrm{d}}{\mathrm{d} u} P_{0}\left[\eta_{(-u, u)}>t\right] \leq(1+\epsilon) \exp \left(-\frac{\pi^{2} t}{8 u^{2}}\right) \frac{\pi t}{u^{3}}
$$

for all $u \leq \sqrt{t / M}$.

Let $C=C(\gamma)$. From the hypothesis choose $K>0$ so large that

$$
u^{-C(1+\epsilon)} \leq P\left(\tau_{D}(z)>u\right) \leq u^{-C(1-\epsilon)} \text { for } u \geq K .
$$

We further assume that $t$ is so large that $K<\sqrt{t / M}$. Now by (4.1), after integration by parts,

$$
\begin{aligned}
P_{z}\left[\tau_{D}\left(Z^{1}\right)>t\right] & =\int_{0}^{\infty}\left(\frac{\mathrm{d}}{\mathrm{d} u} P_{0}\left[\eta_{(-u, u)}>t\right]\right) P\left[\tau_{D}(z)>u\right] \mathrm{d} u \\
& \gtrsim t \int_{K}^{\sqrt{t / M}} \exp \left(-\frac{\pi^{2} t}{8 u^{2}}\right) u^{-(C(1+\epsilon)+3)} \mathrm{d} u
\end{aligned}
$$

and changing variables $u^{-2}=x, \mathrm{~d} u=-1 / 2 x^{-3 / 2} \mathrm{~d} x$ the integral is

$$
\gtrsim t \int_{M / t}^{K^{-2}} \exp \left(-\frac{\pi^{2} t x}{8}\right) x^{C(1+\epsilon) / 2} \mathrm{~d} x
$$


Changing variables, $z=\pi^{2} t x / 8$, the integral is

$$
\gtrsim t^{-C(1+\epsilon) / 2} \int_{\pi^{2} M / 8}^{K^{-2} \pi^{2} t / 8} \mathrm{e}^{-z} z^{C(1+\epsilon) / 2} \mathrm{~d} z
$$

Now since for some $c_{0}>0$,

and

$$
\begin{gathered}
\int_{K^{-2} \pi^{2} t / 8}^{\infty} \mathrm{e}^{-z} z^{C(1+\epsilon) / 2} \mathrm{~d} z \leq \mathrm{e}^{-c_{0} t} \\
\int_{0}^{\pi^{2} M / 8} \mathrm{e}^{-z} z^{C(1+\epsilon) / 2} \mathrm{~d} z<\infty
\end{gathered}
$$

We have

$$
\int_{0}^{\infty} \mathrm{e}^{-z} z^{C(1+\epsilon) / 2} \mathrm{~d} z=\Gamma(1+C(1+\epsilon) / 2)
$$

$$
P_{z}\left[\tau_{D}\left(Z^{1}\right)>t\right] \gtrsim t^{-C(1+\epsilon) / 2}
$$

We now give an upper bound.

$$
\begin{aligned}
P_{z}\left[\tau_{D}\left(Z^{1}\right)>t\right] & =\int_{0}^{\infty} P_{0}\left(\eta_{(-u, u)}>t\right) f(u) \mathrm{d} u \\
& \lesssim \int_{0}^{\sqrt{t / M}} \mathrm{e}^{-\frac{\pi^{2} t}{8 u^{2}}} f(u) \mathrm{d} u+\int_{\sqrt{t / M}}^{\infty} f(u) \mathrm{d} u \\
& \lesssim E\left[\exp \left(-\frac{\pi^{2} t}{8\left(\tau_{D}(z)\right)^{2}}\right)\right]+(\sqrt{t / M})^{-C(1-\epsilon)} \\
& \lesssim t^{-C(1-\epsilon) / 2} .
\end{aligned}
$$

Equation (4.7) follows from Theorem 2.1 and the asymptotics of $\tau_{D}(z)$.

Now from Equations (4.6) and (4.7) we have

$$
t^{-C(1+\epsilon) / 2} \lesssim P_{z}\left[\tau_{D}\left(Z^{1}\right)>t\right] \lesssim t^{-C(1-\epsilon) / 2}
$$

Now taking logarithm of the above inequalities, dividing by $\log t$, letting $\epsilon \rightarrow 0$, we obtain the desired result.

Proof of Theorem 1.3. Let $\epsilon>0$. From Lemma 2.2, choose $M>0$ so large that

$$
(1-\epsilon) \exp \left(-\frac{\pi^{2} t}{8 u^{2}}\right) \frac{\pi t}{u^{3}} \leq \frac{\mathrm{d}}{\mathrm{d} u} P_{0}\left[\eta_{(-u, u)}>t\right] \leq(1+\epsilon) \exp \left(-\frac{\pi^{2} t}{8 u^{2}}\right) \frac{\pi t}{u^{3}}
$$

for all $u \leq \sqrt{t / M}$

Let $C=j_{\nu}^{2}$. Given $\epsilon>0$, choose $K>0$ so large that

$$
\exp \left(-C(1+\epsilon) u(\log u)^{-2 / p}\right) \leq P\left(\tau_{P_{f}}(z)>u\right) \leq \exp \left(-C(1-\epsilon) u(\log u)^{-2 / p}\right)
$$

for $u \geq K$. We further assume that $t$ is so large that $K<\sqrt{t / M}$.

Then, by equations (4.8) and (4.9)

$$
P_{z}\left[\tau_{P_{f}}\left(Z^{1}\right)>t\right] \gtrsim t \int_{K}^{\sqrt{t / M}} u^{-3} \exp \left(-\frac{\pi^{2} t}{8 u^{2}}\right) \exp \left(-C(1+\epsilon) u(\log u)^{-2 / p}\right) \mathrm{d} u
$$


changing variables $u^{-2}=x, \mathrm{~d} u=-1 / 2 x^{-3 / 2} \mathrm{~d} x$ the integral is

$$
\gtrsim t \int_{M / t}^{K^{-2}} \exp \left(-\frac{\pi^{2} t x}{8}\right) \exp \left(-C(1+\epsilon) x^{-1 / 2}\left(\log x^{-1 / 2}\right)^{-2 / p}\right) \mathrm{d} x .
$$

Now we set

$$
v(x)=C(1+\epsilon) x^{-1 / 2}\left(\log x^{-1 / 2}\right)^{-2 / p}
$$

which gives

$$
\mathrm{d} v=C(1+\epsilon) x^{-3 / 2}\left(\log x^{-1 / 2}\right)^{-2 / p}\left[-1 / 2+1 / p\left(\log x^{-1 / 2}\right)^{-1}\right] \mathrm{d} x=-V \mathrm{~d} x .
$$

Then the integral is

$$
\gtrsim t \int_{M / t}^{K^{-2}} V V^{-1} \exp \left(-\frac{\pi^{2} t x}{8}\right) \exp \left(-C(1+\epsilon) x^{-1 / 2}\left(\log x^{-1 / 2}\right)^{-2 / p}\right) \mathrm{d} x .
$$

Now for $x \geq M / t$

$$
V^{-1} \gtrsim t^{-3 / 2}\left[1 / 2-1 / p(\log \sqrt{t / M})^{-1}\right]^{-1} \gtrsim t^{-3 / 2}
$$

Hence the integral is

$$
\gtrsim t^{-1 / 2} \int_{M / t}^{K^{-2}} \exp \left(-\frac{\pi^{2} t x}{8}\right) \exp (-v(x)) V(x) \mathrm{d} x .
$$

Now from de Bruijn's Tauberian Theorem 2.1 we have

$$
\log \int_{0}^{\infty} \exp \left(-\frac{\pi^{2} t x}{8}\right) \exp (-v(x)) V(x) \mathrm{d} x \sim-C(p, \epsilon) t^{1 / 3}(\log t)^{-4 /(3 p)}
$$

where $C(p, \epsilon)=(3 / 2)^{(4+3 p) / 3 p} 2^{1 / 3}\left((1+\epsilon) j_{\nu}^{2} 2^{2 / p}\right)^{2 / 3}\left(\pi^{2} / 8\right)^{1 / 3}$.

For some $c_{1}, c_{2}>0$ we have the following bounds:

$$
\int_{0}^{M / t} \exp \left(-\frac{\pi^{2} t x}{8}\right) \exp (-v(x)) V(x) \mathrm{d} x \leq \mathrm{e}^{-c_{1} t^{1 / 2}(\log t)^{-2 / p}},
$$

and

from which we get

$$
\int_{K^{-2}}^{\infty} \exp \left(-\frac{\pi^{2} t x}{8}\right) \exp (-v(x)) V(x) \mathrm{d} x \leq \mathrm{e}^{-c_{2} t}
$$

$$
P_{z}\left[\tau_{P_{f}}\left(Z^{1}\right)>t\right] \gtrsim \exp \left(-(1+\epsilon)^{2} C(p, \epsilon) t^{1 / 3}(\log t)^{-4 / 3 p}\right) .
$$

We next give the upper bound

$$
\begin{aligned}
P_{z}\left[\tau_{P_{f}}\left(Z^{1}\right)>t\right] & =\int_{0}^{\infty} P_{0}\left(\eta_{(-u, u)}>t\right) f(u) \mathrm{d} u \\
& \lesssim \int_{0}^{\sqrt{t / M}} \mathrm{e}^{-\frac{\pi^{2} t}{8 u^{2}}} f(u) \mathrm{d} u+\int_{\sqrt{t / M}}^{\infty} f(u) \mathrm{d} u \\
& \lesssim E\left[\exp \left(-\frac{\pi^{2} t}{8\left(\tau_{P_{f}}(z)\right)^{2}}\right)\right]+\exp \left(-C(1-\epsilon) t^{1 / 2}(\log t)^{-2 / p}\right) .
\end{aligned}
$$


The upper bound follows from de Bruijn's Tauberian Theorem by observing from (1.5) that as $x \rightarrow 0^{+}$

$$
\log P\left[1 /\left(\tau_{P_{f}}(z)\right)^{2} \leq x\right]=\log P\left[\tau_{P_{f}}(z) \geq x^{-1 / 2}\right] \sim-C x^{-1 / 2}\left(\left|\log x^{-1 / 2}\right|\right)^{-2 / p} .
$$

Hence

$$
P_{z}\left[\tau_{P_{f}}\left(Z^{1}\right)>t\right] \lesssim \exp \left(-(1-\epsilon) D(p, \epsilon) t^{1 / 3}(\log t)^{-4 / 3 p}\right)
$$

where $D(p, \epsilon)=(3 / 2)^{(4+3 p) / 3 p} 2^{1 / 3}\left((1-\epsilon) j_{\nu}^{2} 2^{2 / p}\right)^{2 / 3}\left(\pi^{2} / 8\right)^{1 / 3}$.

Therefore from equations (4.12) and (4.13), we obtain

$$
\begin{aligned}
& \exp \left(-(1+\epsilon)^{2} C(p, \epsilon) t^{1 / 3}(\log t)^{-4 / 3 p}\right) \\
& \lesssim P_{z}\left[\tau_{P_{f}}\left(Z^{1}\right)>t\right] \\
& \lesssim \exp \left(-(1-\epsilon)^{2} D(p, \epsilon) t^{1 / 3}(\log t)^{-4 / 3 p}\right) .
\end{aligned}
$$

Now taking logarithms, multiplying by $\left.t^{-1 / 3}(\log t)^{4 / 3 p}\right)$, letting $t \rightarrow \infty$ and then letting $\epsilon \rightarrow 0$, we get the desired result.

Proof of Theorem 1.4. The proof follows the same steps of the proof of Theorem 1.3, so we omit the details.

Acknowledgements. I would like to thank Professor Rodrigo Bañuelos, my academic advisor, for suggesting this problem to me and for his guidance on this paper.

\section{REFERENCES}

[1] H. Allouba, Brownian-time processes: The pde connection and the corresponding Feynman-Kac formula. Trans. Amer. Math. Soc. 354 (2002) 4627-4637.

[2] H. Allouba and W. Zheng, Brownian-time processes: The pde connection and the half-derivative generator. Ann. Prob. 29 (2001) 1780-1795.

[3] R. Bañuelos and R.D. DeBlassie, The exit distribution for iterated Brownian motion in cones. Stochastic Processes Appl. 116 (2006) 36-69.

[4] R. Bañuelos, R.D. DeBlassie and R. Smits, The first exit time of planar Brownian motion from the interior of a parabola. Ann. Prob. 29 (2001) 882-901.

[5] R. Bañuelos, R. Smits, Brownian motion in cones. Probab. Theory Relat. Fields 108 (1997) 299-319.

[6] N.H. Bingham, C.M. Goldie and J.L. Teugels, Regular Variation. Cambridge University Press, Cambridge (1987).

[7] K. Burdzy, Some path properties of iterated Brownian motion, in Seminar on Stochastic Processes, E. Çinlar, K.L. Chung and M.J. Sharpe, Eds., Birkhäuser, Boston (1993) 67-87.

[8] K. Burdzy, Variation of iterated Brownian motion, in Workshops and Conference on Measure-valued Processes, Stochastic Partial Differential Equations and Interacting Particle Systems, D.A. Dawson, Ed., Amer. Math. Soc. Providence, RI (1994) $35-53$.

[9] K. Burdzy and D. Khoshnevisan, Brownian motion in a Brownian crack. Ann. Appl. Probabl. 8 (1998) 708-748.

[10] E. Csàki, M. Csörgő, A. Földes and P. Révész, The local time of iterated Brownian motion. J. Theoret. Probab. 9 (1996) $717-743$.

[11] R.D. DeBlassie, Exit times from cones in $\mathbb{R}^{n}$ of Brownian motion. Prob. Th. Rel. Fields 74 (1987) 1-29.

[12] R.D. DeBlassie, Iterated Brownian motion in an open set. Ann. Appl. Prob. 14 (2004) 1529-1558.

[13] R.D. DeBlassie and R. Smits, Brownian motion in twisted domains. Trans. Amer. Math. Soc. 357 (2005) 1245-1274.

[14] N.G. De Bruijn, Asymptotic methods in analysis. North-Holland Publishing Co., Amsterdam (1957).

[15] N. Eisenbum and Z. Shi, Uniform oscillations of the local time of iterated Brownian motion. Bernoulli 5 (1999) 49-65.

[16] W. Feller, An Introduction to Probability Theory and its Applications. Wiley, New York (1971).

[17] Y. Kasahara, Tauberian theorems of exponential type. J. Math. Kyoto Univ. 12 (1978) 209-219.

[18] D. Khoshnevisan and T.M. Lewis, Stochastic calculus for Brownian motion in a Brownian fracture. Ann. Applied Probabl. 9 (1999) 629-667.

[19] D. Khoshnevisan and T.M. Lewis, Chung's law of the iterated logarithm for iterated Brownian motion. Ann. Inst. H. Poincaré Probab. Statist. 32 (1996) 349-359.

[20] O. Laporte, Absorption coefficients for thermal neutrons. Phys. Rev. 52 (1937) 72-74.

[21] W. Li, The first exit time of a Brownian motion from an unbounded convex domain. Ann. Probab. 31 (2003) 1078-1096.

[22] M. Lifshits and Z. Shi, The first exit time of Brownian motion from a parabolic domain. Bernoulli 8 (2002) 745-765. 
[23] E. Nane, Iterated Brownian motion in parabola-shaped domains. Potential Analysis 24 (2006) 105-123.

[24] E. Nane, Iterated Brownian motion in bounded domains in $\mathbb{R}^{n}$. Stochastic Processes Appl. 116 (2006) 905-916.

[25] E. Nane, Higher order PDE's and iterated processes. Accepted Trans. Amer. Math. Soc. math.PR/0508262.

[26] E. Nane, Laws of the iterated logarithm for $\alpha$-time Brownian motion. Electron. J. Probab. 11 (2006) $34-459$ (electronic).

[27] E. Nane, Isoperimetric-type inequalities for iterated Brownian motion in $\mathbb{R}^{n}$. Submitted, math.PR/0602188.

[28] S.C. Port and C.J. Stone, Brownian motion and Classical potential theory. Academic, New York (1978).

[29] Y. Xiao, Local times and related properties of multidimensional iterated Brownian motion. J. Theoret. Probab. 11 (1998) $383-408$. 